\title{
Integrability of the generalised Hill problem
}

\author{
Thierry Combot • Andrzej J. Maciejewski • \\ Maria Przybylska 1 D
}

Received: 4 May 2021 / Accepted: 29 October 2021 / Published online: 7 December 2021

(C) The Author(s) 2021

\begin{abstract}
We consider a certain two-parameter generalisation of the planar Hill lunar problem. We prove that for nonzero values of these parameters the system is not integrable in the Liouville sense. For special choices of parameters the system coincides with the classical Hill system, the integrable synodical Kepler problem or the integrable parametric Hénon system. We prove that the synodical Kepler problem is not super-integrable, and that the parametric Hénon problem is super-integrable for infinitely many values of the parameter.
\end{abstract}

Keywords The Hill problem · Integrability obstructions $\cdot$ Super-integrability $\cdot$ Regularisation

\section{Introduction}

In this paper, we consider a certain version of the planar circular Hill problem. In the classical formulation the Hill problem is a limiting case of the restricted three

T. Combot

Université de Burgogne, 9 Avenue A. Savary, BP 47870,

21078 Dijon Cedex, France

e-mail: thierry.combot@u-bourgogne.fr

A. J. Maciejewski

Janusz Gil Institute of Astronomy, University of Zielona

Góra, Licealna 9, 65-417 Zielona Góra, Poland

e-mail: a.maciejewski@ia.uz.zgora.pl

M. Przybylska $(\varangle)$

Institute of Physics, University of Zielona Góra, Licealna

9, 65-417 Zielona Góra, Poland

e-mail: m.przybylska@if.uz.zgora.pl body problem convenient for studying dynamics of a massless body in the vicinity of smaller primary [1].

The Hill problem was developed by George Hill, see [2] in order to construct the theory of motion of the Moon in the Sun-Earth-Moon system. Later several versions of this problem were considered. Application of the Hill problem with appropriate modifications in the stellar dynamics is discussed in [3]. A pseudoNewtonian Hill problem was investigated in [4-6]. In this model the Newtonian potential is replaced by the Paczyński-Wiita potential [7]. It can be considered as the zeroth order of the general relativistic Hill problem.

A quite general method of Hill's type approximation of the equations of motion is described in [8]. Yet another generalisation of the Hill problem was considered in [9]. It is called the photo-gravitational Hill problem, which incorporates an influence of the radiation pressure forces on the motion of the massless body.

Intensive numerical investigations of the classical Hill problem were performed in [10]. Among other things the author gives a classification of orbits in the considered problem.

In this paper, we consider a generalised Hill problem proposed by [11,12] and described by the following Hamiltonian function

$$
\begin{aligned}
H= & \frac{1}{2}\left(p_{x}^{2}+p_{y}^{2}\right)+y p_{x}-x p_{y}+\frac{\sigma}{|\boldsymbol{r}|} \\
& +\varepsilon\left(-x^{2}+\frac{1}{2} y^{2}\right),
\end{aligned}
$$


where $\boldsymbol{r}=(x, y), \boldsymbol{p}_{\boldsymbol{r}}=\left(p_{x}, p_{y}\right),|\boldsymbol{r}|=\sqrt{x^{2}+y^{2}}$, and $\sigma$ and $\varepsilon$ are real parameters. This generalisation allows to study in a uniform manner a variety of systems, among which the following are included

- For $(\sigma, \varepsilon)=(-1,1)$ - the classical Hill problem, see e.g. [1,3];

- For $(\sigma, \varepsilon)=(1,1)$ - the anti-Hill problem, see [11-13];

- For $(\sigma, \varepsilon)=(-1,0)$ - the synodical Kepler problem, see e.g. Chapter III in [14];

- For $(\sigma, \varepsilon)=(\sigma, 0)$, where $\sigma \in \mathbb{R} \backslash\{0\}$ - the parametric synodical Kepler problem;

- For $(\sigma, \varepsilon)=(0,1)$ - the Hénon problem, see [1517];

- For $(\sigma, \varepsilon)=(0, \varepsilon)$, where $\varepsilon \in \mathbb{R}$ - the parametric Hénon problem, see [11,17];

- For $\varepsilon \neq 0$ and $\sigma \neq 0$ - a special case of the photogravitational Hill problem [9].

The other versions of the Hill problem mentioned earlier are not covered by the model defined by Hamiltonian (1).

The aim of this paper is to study integrability in the Liouville sense of the two-parameter family of Hamiltonian systems (1). Also general properties of this system such as equilibria and qualitative behaviour of phase curves illustrated by the Poincaré cross sections for various values of parameters are analysed.

Integrability of two among of these systems: the synodical Kepler problem and the Hénon problem is well known. In the synodical Kepler problem the additional first integral is the angular momentum component $F=x p_{y}-y p_{x}$ and this function remains the first integral also for the parametric synodical Kepler problem. For the Hénon problem the additional first integral takes the form

$F=p_{x}^{2}+2\left(p_{x} y-2 p_{y} x\right)-3 x^{2}+y^{2}$.

One can easily check that this first integral generalises to

$$
\begin{aligned}
F= & 3 p_{x}^{2} \varepsilon+2(2+\varepsilon) p_{x} y-4(1+2 \varepsilon) p_{y} x \\
& +3 \varepsilon\left(y^{2}-x^{2}(1+2 \varepsilon)\right)
\end{aligned}
$$

for the parametric Hénon system. On the other hand the non-integrability of the classical Hill problem was proved in [18].

We complement these results by proving that Hamiltonian equations generated by the Hamilton function (1) for $\sigma \varepsilon \neq 0$ are non-integrable. In particular, we give a simpler non-integrability proof for the classical Hill problem. As result we can formulate necessary and sufficient integrability conditions for this two parameter family of Hamiltonian systems

Theorem 1 The Hamiltonian system (1) is integrable in the Liouville sense in the class of rational functions of variables $x, y, p_{x}, p_{y}, r=|\boldsymbol{r}|$ if and only if $\sigma \varepsilon=0$.

Moreover, we analyse super-integrability of the parametric Hénon system and the synodical Kepler problem. We show that the parametric Hénon system for infinitely many rational values of parameter $\varepsilon$ appears to be super-integrable and this problem is discussed in Sect. 5.1. In Sect. 5.2, we explain that the synodical Kepler problem is not super-integrable.

In this paper, we study integrability of the system in the framework of the differential Galois theory. For Hamiltonian systems the necessary integrability condition is given by the following theorem.

Theorem 2 Assume that a complex Hamiltonian system is meromorphically integrable in the Liouville sense in a neighbourhood of a phase curve $\Gamma$. Then, the identity component of the differential Galois group of the variational equations along $\Gamma$ is Abelian.

For details see [19]. Practical introduction and numerous applications can be found in [20]. To apply the above theorem in Sects. 3 and 4, we consider the complexification of the system defined by Hamiltonian (1).

The plan of the paper is following. In Sect. 2, general properties of the Hamiltonian system (1) are analysed. Equilibria are calculated and their stability in linear approximation is determined. Generic behaviour of phase curves is illustrated by means of Poincaré cross sections presented in two- and three- dimensional spaces. The difficulty of making flat two-dimensional cross sections in cases when cross section surfaces have non-trivial geometry is discussed. In Sect. 3, we derive the regularised Hamiltonian with removed singularity in the origin and we discuss relations between first integral of original and regularised Hamiltonians. Section 4 contains the non-integrability proof of the generalised Hill's problem for the case $\sigma \varepsilon \neq 0$. In the last Sect. 5 , the problem of existence of one more functionally independent first integral for the integrable parametric Hénon system and the synodical Kepler problem is analysed. 


\section{General properties of the dynamics of the system}

Hamilton equations determined by the Hamilton function (1) have the form

$$
\begin{aligned}
& \dot{x}=p_{x}+y, \quad \dot{p}_{x}=p_{y}+2 \varepsilon x+\frac{\sigma x}{\left(x^{2}+y^{2}\right)^{3 / 2}}, \\
& \dot{y}=p_{y}-x, \quad \dot{p}_{y}=-p_{x}-\varepsilon y+\frac{\sigma y}{\left(x^{2}+y^{2}\right)^{3 / 2}} .
\end{aligned}
$$

We determine real equilibria and their types for this system. Clearly, if $\left(x, y, p_{x}, p_{y}\right) \in \mathbb{R}^{4}$ is an equilibrium, then $p_{x}=-y$ and $p_{y}=x$. Using this fact we deduce that either $x=0$ whence $p_{x}=-y, p_{y}=0$ and $y$ is a real solution of the equation

$$
\frac{\sigma}{\left(y^{2}\right)^{\frac{3}{2}}}=\varepsilon-1 \text {, }
$$

or $y=0$ whence $p_{x}=0, p_{y}=x$ and $x$ is a real solution of the equation

$$
\frac{\sigma}{\left(x^{2}\right)^{\frac{3}{2}}}=-1-2 \varepsilon \text {. }
$$

The type of an equilibrium is determined by the eigenvalues of the Jacobian matrix

$$
\mathbb{L}=\left[\begin{array}{llll}
0 & 1 & 1 & 0 \\
-1 & 0 & 0 & 1 \\
2 \varepsilon+\frac{\left(y^{2}-2 x^{2}\right) \sigma}{\left(x^{2}+y^{2}\right)^{5 / 2}} & -\frac{3 x y \sigma}{\left(x^{2}+y^{2}\right)^{5 / 2}} & 0 & 1 \\
-\frac{3 x y \sigma}{\left(x^{2}+y^{2}\right)^{5 / 2}} & \frac{\left(x^{2}-2 y^{2}\right) \sigma}{\left(x^{2}+y^{2}\right)^{5 / 2}}-\varepsilon & -1 & 0
\end{array}\right]
$$

of the right-hand sides of Hamilton's Eq. (3) evaluated at this equilibrium.

In our further analysis we assume that $\sigma \neq 0$. An analysis of the conditions given above leads to two pairs of isolated equilibria. The first pair of equilibria $S_{ \pm}$ lying on $y$-axis is given by

$x=0, \quad y= \pm \sqrt[3]{\frac{\sigma}{\varepsilon-1}}, \quad p_{x}=\mp \sqrt[3]{\frac{\sigma}{\varepsilon-1}}, \quad p_{y}=0$.

It exists iff $\sigma(\varepsilon-1)>0$. Eigenvalues of matrix $\mathbb{L}$ are

$$
\begin{aligned}
& \lambda_{1,2}= \pm \mathrm{i} \frac{\sqrt{1+\sqrt{36 \varepsilon(\varepsilon-1)+1}}}{\sqrt{2}}, \\
& \lambda_{3,4}=\frac{\sqrt{-1+\sqrt{36 \varepsilon(\varepsilon-1)+1}}}{\sqrt{2}} .
\end{aligned}
$$

For $\varepsilon>1$ and $\sigma>0$ these equilibria are always saddlecentre. If $\varepsilon<1$ and $\sigma<0$, then character of equilibria depends on $\varepsilon$. Namely, if $\frac{1}{6}(3-2 \sqrt{2})<\varepsilon<\frac{1}{6}(3+$ $2 \sqrt{2})$, then $36 \varepsilon(\varepsilon-1)+1<0$ and all eigenvalues have real and imaginary parts different from zero

$$
\begin{aligned}
\lambda_{1,2}= & \pm \frac{1}{2}[-\sqrt{-1+\sqrt{36 \varepsilon(1-\varepsilon)}} \\
& +i \sqrt{1+\sqrt{36 \varepsilon(1-\varepsilon)}],} \\
\lambda_{3,4}= & \pm \frac{1}{2}[\sqrt{-1+\sqrt{36 \varepsilon(1-\varepsilon)}} \\
& +i \sqrt{1+\sqrt{36 \varepsilon(1-\varepsilon)}}],
\end{aligned}
$$

thus $S_{ \pm}$are focus-focus. If $\varepsilon \in\left(0, \frac{1}{6}(3-2 \sqrt{2}] \cup\right.$ $\left[\frac{1}{6}(3+2 \sqrt{2}), 1\right)$, then $1>\sqrt{36 \varepsilon(\varepsilon-1)+1}>0$, thus eigenvalues $\lambda_{3,4}$ in (7) become also purely imaginary numbers and $S_{ \pm}$become centre-centre. If $\varepsilon=$ $\frac{1}{6}(3-2 \sqrt{2})$ or $\varepsilon=\frac{1}{6}(3+2 \sqrt{2})$, the matrix $\mathbb{L}$ has multiple eigenvalues $\pm \mathrm{i} \frac{\sqrt{2}}{2}$ with two-dimensional Jordan blocks. Finally, for arbitrary $\varepsilon<0$ equilibria $S_{ \pm}$are saddle-centre.

The second pair of equilibria $L_{ \pm}$lying on the $x$-axis is given by

$$
\begin{aligned}
x & = \pm \sqrt[3]{-\frac{\sigma}{2 \varepsilon+1}}, \quad y=0, \quad p_{x}=0, \\
p_{y} & = \pm \sqrt[3]{-\frac{\sigma}{2 \varepsilon+1}} .
\end{aligned}
$$

It exists iff $\sigma(2 \varepsilon+1)<0$. Eigenvalues of matrix $\mathbb{L}$ at these equilibria are

$$
\begin{aligned}
& \lambda_{1,2}= \pm \mathrm{i} \frac{\sqrt{1-3 \varepsilon+\sqrt{81 \varepsilon^{2}+30 \varepsilon+1}}}{\sqrt{2}}, \\
& \lambda_{3,4}= \pm \frac{\sqrt{3 \varepsilon-1+\sqrt{81 \varepsilon^{2}+30 \varepsilon+1}}}{\sqrt{2}} .
\end{aligned}
$$

If $\varepsilon<-1 / 2$ and $\sigma>0$, then these points are saddlecentre. If $\varepsilon>-1 / 2$ and $\sigma<0$, then character of the equilibria depends on $\varepsilon$. In the interval $-\frac{1}{3}<\varepsilon<-\frac{1}{27}$ we have $81 \varepsilon^{2}+30 \varepsilon+1<0$, and so, all eigenvalues

$$
\begin{aligned}
\lambda_{1,2}= & \pm \frac{1}{2}[-\sqrt{3 \varepsilon-1+\sqrt{-36 \varepsilon(1+2 \varepsilon)}} \\
& +i \sqrt{1-3 \varepsilon+\sqrt{-36 \varepsilon(1+2 \varepsilon)}}], \\
\lambda_{3,4}= & \pm \frac{1}{2}[\sqrt{3 \varepsilon-1+\sqrt{-36 \varepsilon(1+2 \varepsilon)}} \\
& +i \sqrt{1-3 \varepsilon+\sqrt{-36 \varepsilon(1+2 \varepsilon)}}],
\end{aligned}
$$

have real and imaginary parts different from zero, thus $L_{ \pm}$are focus-focus equilibria. For $\varepsilon \in\left(-\frac{1}{2},-\frac{1}{3}\right] \cup$ 
Fig. 1 Character of isolated equilibria $S_{ \pm}$and $L_{ \pm}$. Notation:

$\mathrm{C}-\mathrm{C}$-centre-centre, $\mathrm{S}-\mathrm{C}$ - saddle-centre, F-F-focus-focus

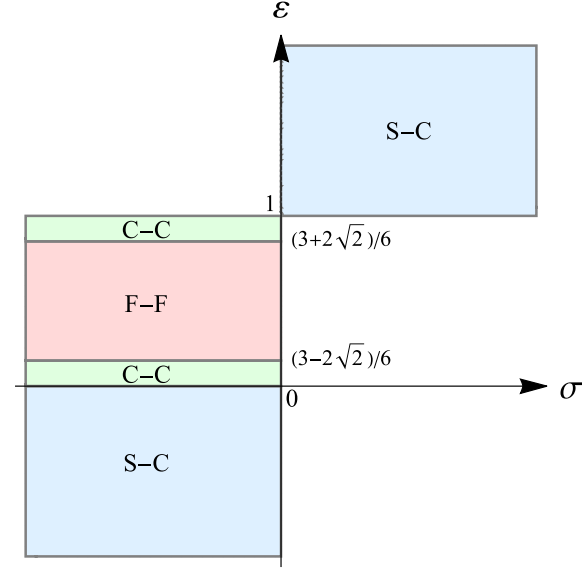

(a) equilibria $S_{ \pm}$

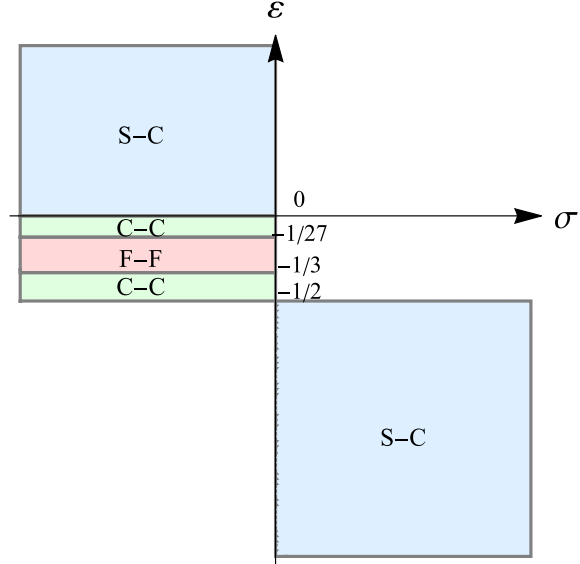

(b) equilibria $L_{ \pm}$ $\left[-\frac{1}{27}, 0\right)$ although $81 \varepsilon^{2}+30 \varepsilon+1>0$, we have $3 \varepsilon-1+\sqrt{81 \varepsilon^{2}+30 \varepsilon+1}<0$, thus eigenvalues $\lambda_{3,4}$ become purely imaginary numbers and equilibria $L_{ \pm}$become centre-centre. For $\varepsilon=-\frac{1}{27}$ matrix $\mathbb{L}$ has multiple eigenvalues $\pm \mathrm{i} \frac{\sqrt{5}}{3}$ and for $\varepsilon=-\frac{1}{3}$ multiple eigenvalues $\pm \mathrm{i}$ with 2-dimensional Jordan blocks. For arbitrary $\varepsilon>0$ equilibria $L_{ \pm}$become saddle-centres. Character of equilibria on the plane of parameters $(\sigma, \varepsilon)$ is shown in Fig.1.

Let us notice that for $\sigma<0$ and $-\frac{1}{2}<\varepsilon<1$ the generalised Hill system has four isolated equilibria $S_{ \pm}, L_{ \pm}$. For $\sigma>0$ and $-\frac{1}{2}<\varepsilon<1$ the generalised Hill problem has no equilibrium.

For $\varepsilon=0$ we obtain the parametric synodical Kepler problem with infinitely many equilibria for $\sigma<0$ :

$p_{x}=-y, \quad p_{y}=x, \quad$ and $(x, y) \neq(0,0)$

satisfies $\left(x^{2}+y^{2}\right)^{3 / 2}+\sigma=0$, with eigenvalues of $\mathbb{L}$ equal to $\lambda_{1}=\lambda_{2}=0, \lambda_{3,4}= \pm \mathrm{i}$. For $\varepsilon=0$ and $\sigma>0$ the parametric synodical Kepler problem has no real equilibria solutions.

The necessary conditions for linear stability are as follows. If $\sigma<0$, then

$$
\begin{aligned}
& \varepsilon \in\left(-\frac{1}{2},-\frac{1}{3}\right) \cup\left(-\frac{1}{27}, \frac{1}{6}(3-2 \sqrt{2})\right) \\
& \cup\left(\frac{1}{6}(3+2 \sqrt{2}), 1\right) .
\end{aligned}
$$

For $\sigma>0$ the equilibria are linearly stable if $\varepsilon=0$.

To visualise behaviour of trajectories the Poincaré cross section technique is frequently used. We select an energy level

$M_{E}:=\left\{\left(x, y, p_{x}, p_{y}\right) \in \mathbb{R}^{4} \mid H\left(x, y, p_{x}, p_{y}\right)=E\right\}$.

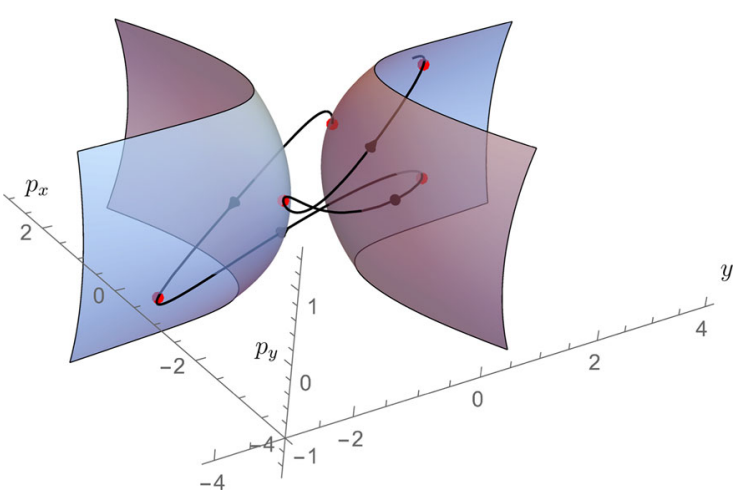

Fig. 2 A trajectory in the energy level $M_{E}$ with $E=2, \sigma=1$ and $\varepsilon=-\frac{1}{4}$. Two components of the cross section surface $x=0$ on this energy level are shown. Intersections of the trajectory with the cross section surface are marked by red dots

It is a three-dimensional manifold. Next, we have to choose a cross section surface $\mathcal{C}$ in $M_{E}$. We require that in a certain domain on this surface phase trajectories intersect it transversally, and, moreover, through each point in the chosen domain passes exactly one trajectory. In order to get a clear graphical representation, we would like also to represent the domain with marked intersection points as a domain in a plane. Customary as a cross section plane $x=0$ (or $y=0$ ) is taken. Moreover, as coordinates on this surface in $M_{E}$ a pair of conjugated canonical variables: a coordinate and the corresponding momentum is chosen. This choice is good provided that the projection of the chosen part of the cross section surface on the canonical plane is one to one. 


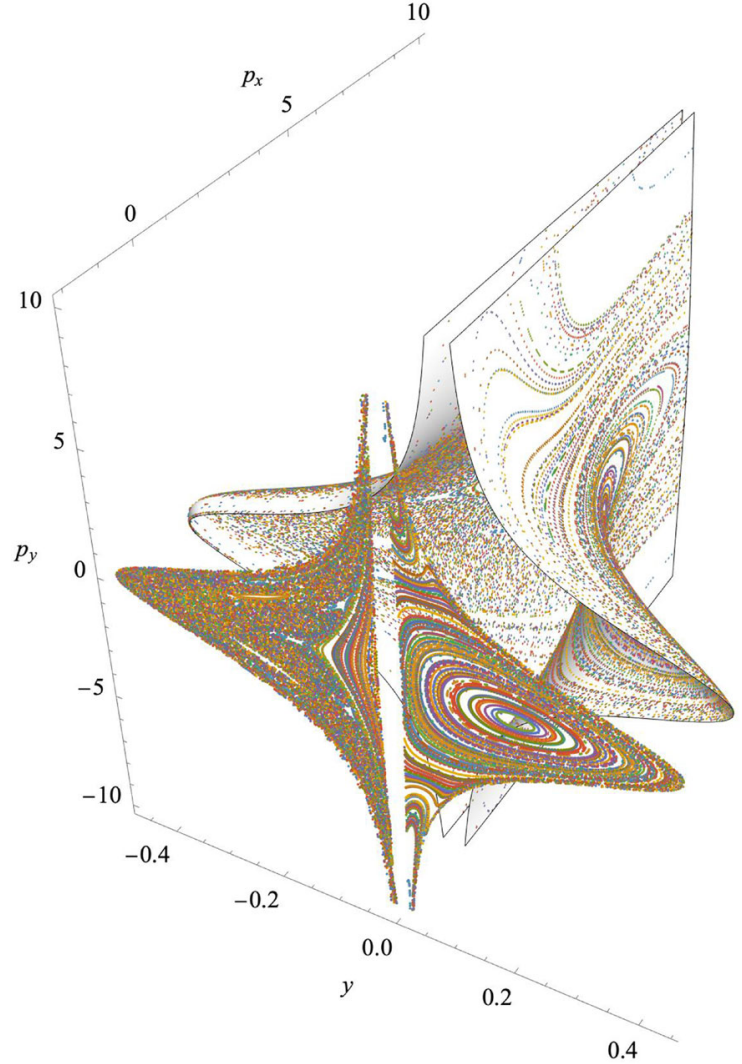

Fig. 3 Energy level $M_{E}$ for $E=-\frac{3 \sqrt[3]{3}}{2} \approx-2.16337, \sigma=-1$ and $\varepsilon=1$ with the part of the cross section surface with $p_{x}>0$. The cross section points for more than 200 orbits are shown

For our first numerical experiments we took $\left(y, p_{x}\right.$, $p_{y}$ ) as coordinates on $M_{E}$ and cross section surface $\mathcal{C}$ is defined by intersection of $M_{E}$ with plane $x=0$. An example is shown in Fig. 2. Clearly, the cross section surface $\mathcal{C}$ has two components. With our choices of coordinates on $M_{E}$ these components are given by

$$
\begin{aligned}
\mathcal{C}_{+}= & \left\{\left(y, p_{x}, p_{y}\right) \in \mathbb{R}^{3} \mid y\left(p_{x}^{2}+p_{y}^{2}+2 p_{x} y\right.\right. \\
& \left.\left.+y^{2} \varepsilon-2 E\right)+2 \sigma=0, y>0\right\}, \\
\mathcal{C}_{-}= & \left\{\left(y, p_{x}, p_{y}\right) \in \mathbb{R}^{3} \mid y\left(p_{x}^{2}+p_{y}^{2}+2 p_{x} y\right.\right. \\
& \left.\left.+y^{2} \varepsilon-2 E\right)-2 \sigma=0, y<0\right\} .
\end{aligned}
$$

The considered system has symplectic symmetry $\left(x, y, p_{x}, p_{y}\right) \mapsto-\left(x, y, p_{x}, p_{y}\right)$. Thus, if $\left(y, p_{x}, p_{y}\right)$ $\in \mathcal{C}$, then also $\left(-y,-p_{x},-p_{y}\right) \in \mathcal{C}$. This is why, without loss of the generality, we can consider only one part of the cross section specified by the sign of one coordinate. In all cases with $\sigma<0$ we take part of the cross section surface with $p_{x}>0$. An example shown in Fig. 3 has the cross section with two com- ponents and both of them have one to one projection onto $\left(y, p_{y}\right)$-plane. The points of the cross section surface are generated for more than 200 orbits. Hereinafter cross sections are presented in this plane. Figs. 4, 5 and 6 illustrate dynamics for $\sigma=-1$, for positive or negative values of $\varepsilon \neq 1$ and for two values of energy. It is visible that the dynamics of the system with lower energy is quite regular while in the cross sections that correspond to higher energy values chaotic zones are clearly visible.

The main difficulty in generation of cross sections in the considered system is the fact that energy levels are not compact and there are orbits which escape or which have only a few intersections with the cross section surface. For cases with $\sigma<0$ the origin is an attracting centre, so we can consider system as a perturbation of the Kepler problem and we investigated negative energy levels for arbitrary values of $\varepsilon$. However, if $\sigma>$ 0 , then the centre in origin is repulsive and it is really difficult to obtain a cross section. For these cases we select $\varepsilon$ in such a way that linear part of the vector field generated by Hamiltonian (1) is stable. It appears this is the case if and only if $\varepsilon \in(-1 / 2,0) \cup(8 / 9,1)$. If we select $\varepsilon$ from the prescribed intervals, then it is possible to find orbits which have multiple intersections with the cross section surface.

However, the other problem appears. As it is shown in Fig. 7, cross section points near origin and on the folded part at the front do not project one to one onto the $\left(y, p_{y}\right)$ plane.

This problem can be solved in many ways. The best results we obtained by just passing to variables $\left(x, y, v_{x}, v_{y}\right)$, where $v_{x}=\dot{x}$ and $v_{y}=\dot{y}$. Again as a cross section plane we take $x=0$ and $\left(y, v_{x}, v_{y}\right)$ as coordinates on the energy level $M_{E}$. Now the energy integral reads

$$
\begin{aligned}
E= & \frac{1}{2}\left[v_{x}^{2}+v_{y}^{2}-(1+2 \varepsilon) x^{2}-(1-\varepsilon) y^{2}\right] \\
& +\frac{\sigma}{\sqrt{x^{2}+y^{2}}} .
\end{aligned}
$$

Hence, components $\mathcal{C}_{ \pm}$of the cross section surface $\mathcal{C} \subset M_{E}$ are
$\mathcal{C}_{ \pm}=\left\{\left(y, v_{x}, v_{y}\right) \in \mathbb{R}^{3} \mid v_{x}^{2}+v_{y}^{2}=2 E+(1-\varepsilon) y^{2} \mp \frac{2 \sigma}{y}\right\}$.

Because of the above symmetry of the system, if $\left(y, v_{x}, v_{y}\right) \in \mathcal{C}_{+}$, then $\left(-y,-v_{x},-v_{y}\right) \in \mathcal{C}_{-}$. Thus, one can restrict to $e_{+}$only which is a cone and $y$ is its symmetry axis. Hence points of $\mathcal{C}_{+}$have unique projections onto $\left(v_{x}, v_{y}\right)$ plane. Examples of Poincaré cross 


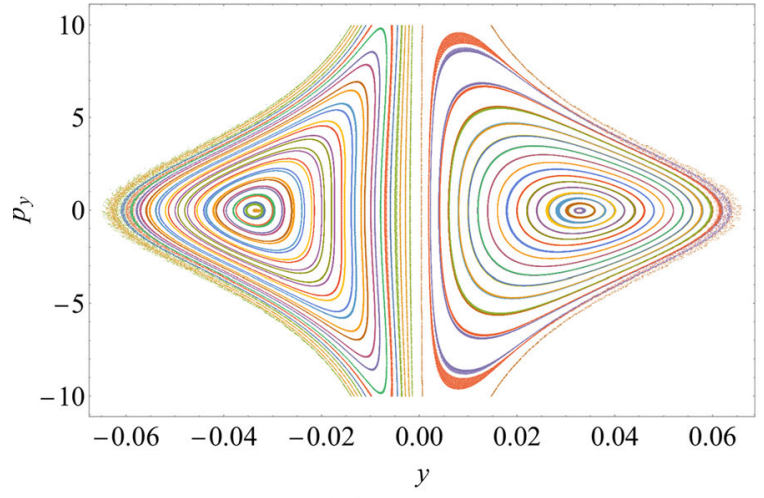

(a) $E=-15$

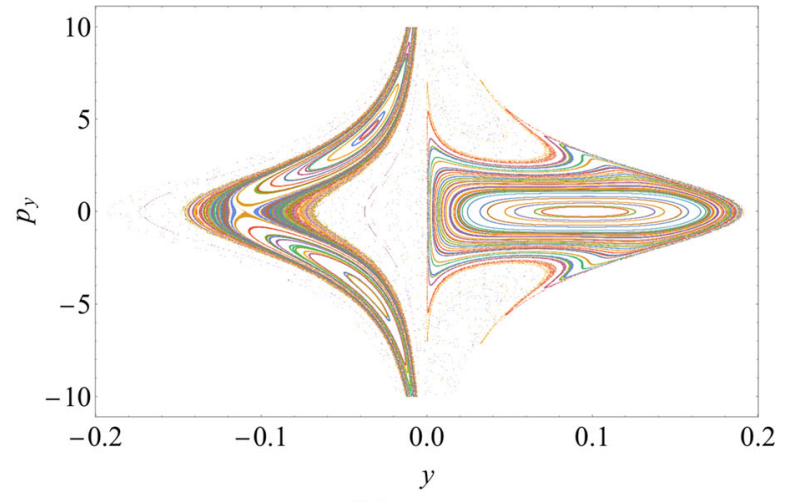

(b) $E=-5$

Fig. 4 Poincaré cross sections for $\sigma=-1$ and $\varepsilon=10$

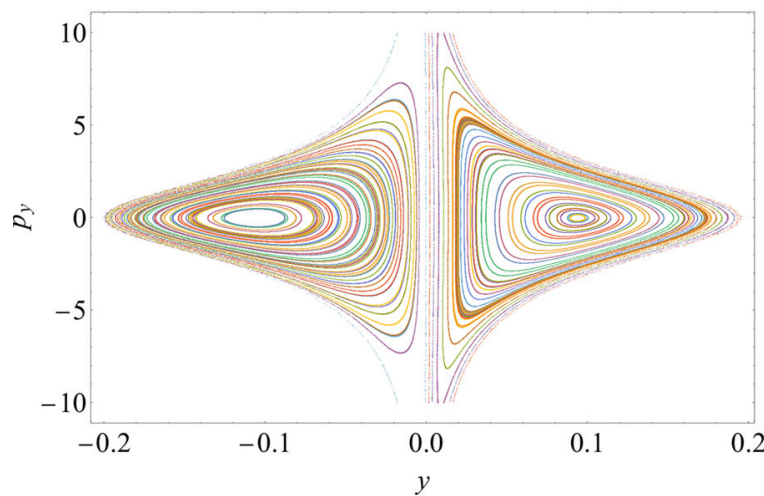

(a) $E=-5$

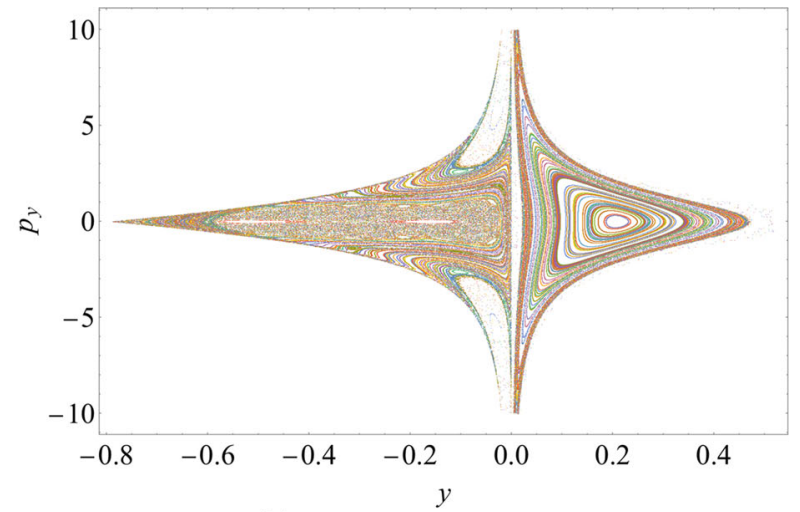

(b) $E=-\frac{3}{2^{2 / 3}} \approx-1.88988$

Fig. 5 Poincaré cross sections for $\sigma=-1$ and $\varepsilon=-1$

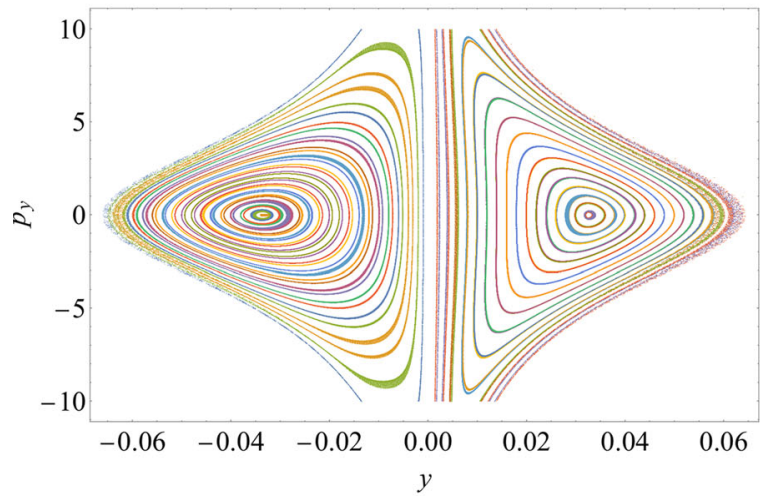

(a) $E=-15$

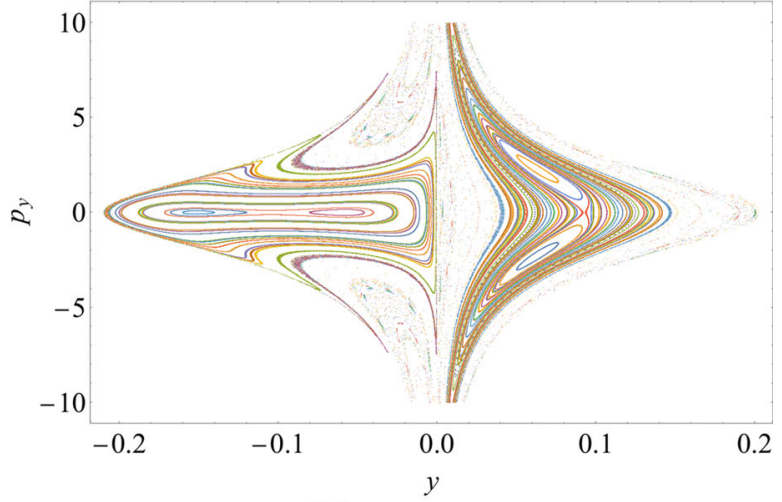

(b) $E=-5$

Fig. 6 Poincaré cross sections for $\sigma=-1$ and $\varepsilon=-10$ 


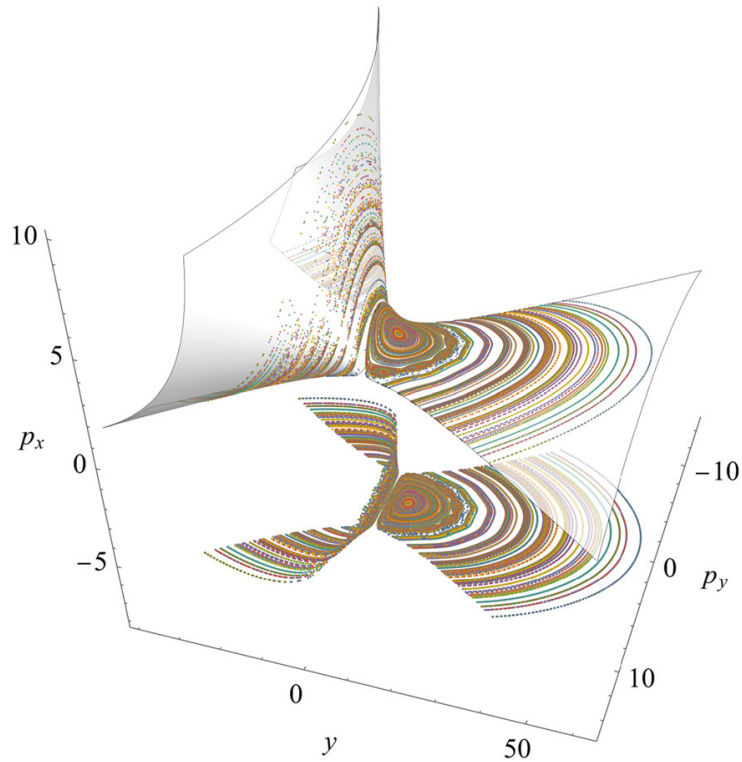

Fig. 7 Energy level $M_{E}$ for $E=5$, parameters $\sigma=1$ and $\varepsilon=-\frac{1}{20}$ with part of the cross section surface with $p_{x}>0$. The cross section points for more than 300 orbits are shown

sections in space $\left(y, v_{x}, v_{y}\right)$ with the corresponding projections onto the plane $\left(v_{x}, v_{y}\right)$ are shown in Figs. 8 and 9. In the central part of the cross section presented in Fig. 8 one can see an island of regular motion around a stable periodic solution surrounded by a large chaotic zone. Around this zone there are regular regions which correspond to quasi-periodic solutions near a stable resonant orbit. The structure of the phase space shown in the cross-section presented in Fig. 9 is different. In the central part there are five very small regular islands corresponding to a periodic solution. Around each of these islands one can see smaller ones which correspond to higher resonant orbits. All this is surrounded by a starlike chaotic zone. Outside this zone there is a large region of regular motions consisting of quasi-periodic orbits near a resonant period solution.

\section{Regularised equations of motion}

The Hamiltonian of the generalised Hill problem with $\sigma \neq 0$ is ramified because of $\sqrt{x^{2}+y^{2}}$ appearing in it. Introducing $r$ such that $r^{2}=x^{2}+y^{2}$, Hamiltonian (1) is now given by

$$
\begin{aligned}
H= & \frac{1}{2}\left(p_{x}^{2}+p_{y}^{2}\right)+y p_{x}-x p_{y}+\frac{\sigma}{r} \\
& +\varepsilon\left(-x^{2}+\frac{1}{2} y^{2}\right),
\end{aligned}
$$

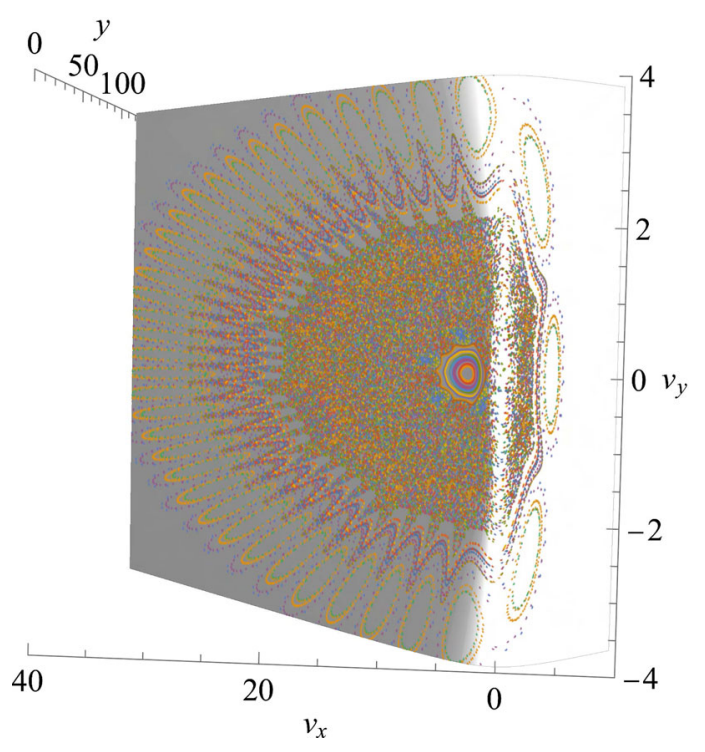

(a) cross-section in $M_{E}$

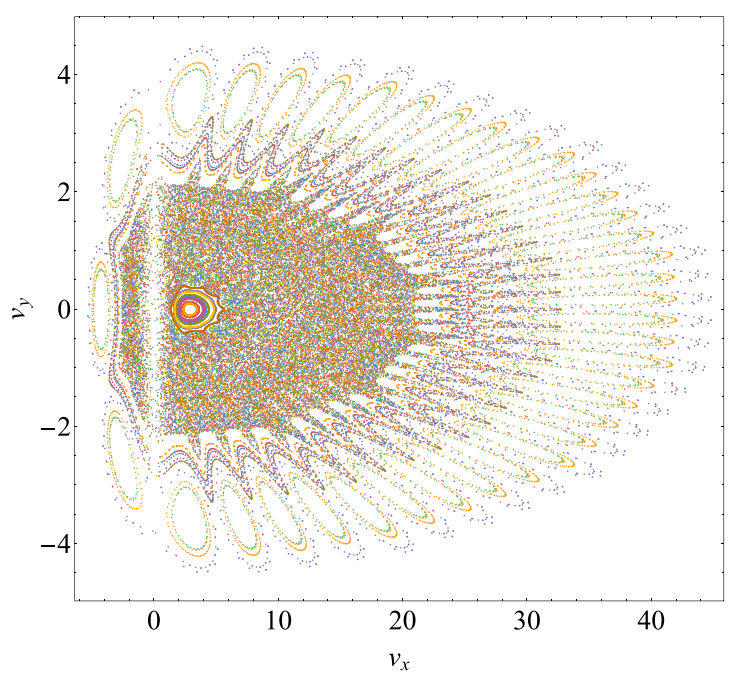

(b) projection into the plane $\left(v_{x}, v_{y}\right)$

Fig. 8 Poincaré cross section for $\sigma=1$ and $\varepsilon=\frac{9}{10}$ on energy level $E=1$

and it is rational on the symplectic manifold $\left\{\left(x, y, p_{x}\right.\right.$, $\left.\left.p_{y}, r\right), \quad r^{2}=x^{2}+y^{2}\right\}$ which is a double cover of $\mathbb{C}^{4}$. The system is singular at the origin; however it is possible to remove the singularity by means of the Levi-Civita regularisation. The following procedure is a modification of that presented in [12]. More details about regularisation procedure one can find in review articles [21,22]. We now consider the coordi- 


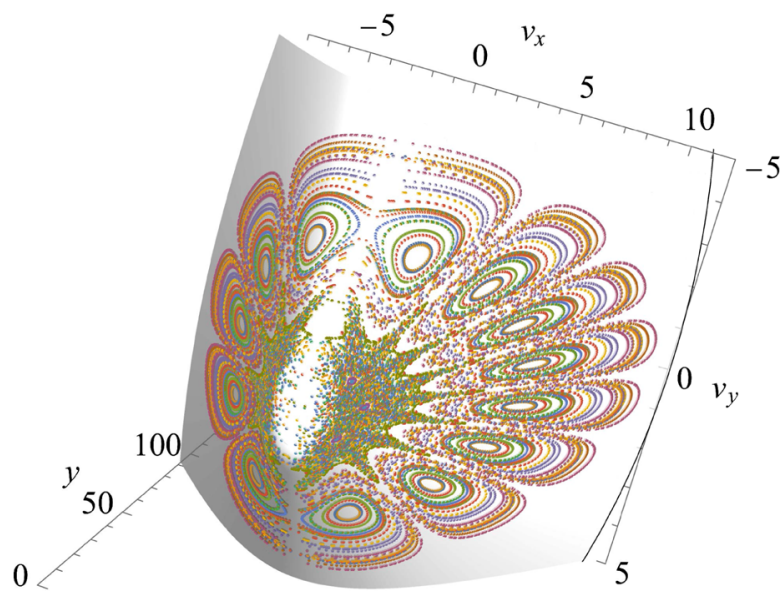

(a) cross-section in $M_{E}$

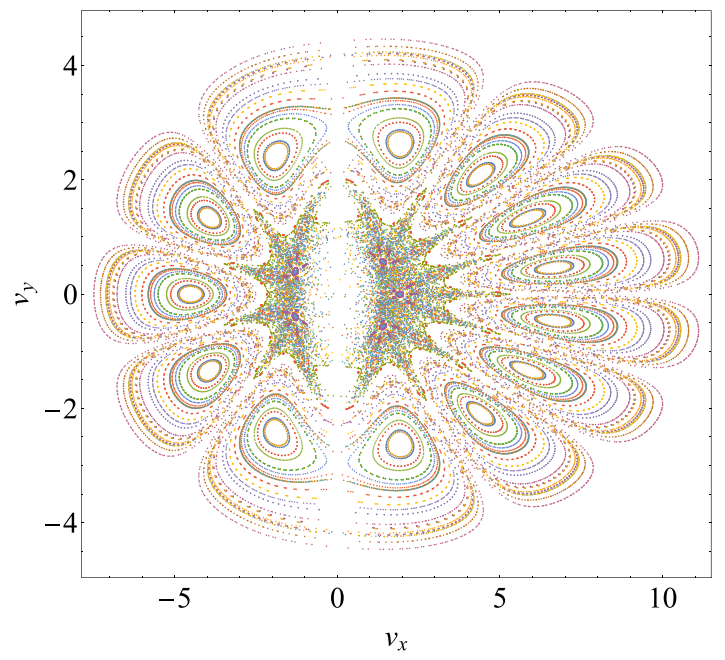

(b) projection into the plane $\left(v_{x}, v_{y}\right)$

Fig. 9 Poincaré cross section for $\sigma=1$ and $\varepsilon=\frac{99}{100}$ on energy level $E=1$

nates change (depending on a parameter $\alpha$ )

$$
\begin{gathered}
x=\frac{u_{1}^{2}-u_{2}^{2}}{\alpha}, \quad y=\frac{2 u_{1} u_{2}}{\alpha}, \quad r=\frac{u_{1}^{2}+u_{2}^{2}}{\alpha}, \\
p_{x}=\frac{u_{1} v_{1}-u_{2} v_{2}}{2 \alpha\left(u_{1}^{2}+u_{2}^{2}\right)}, \quad p_{y}=\frac{u_{1} v_{2}+u_{2} v_{1}}{2 \alpha\left(u_{1}^{2}+u_{2}^{2}\right)} .
\end{gathered}
$$

This coordinates change is projectively symplectic, i.e. it multiplies the symplectic form by $\alpha^{-2}$, and thus it transforms our Hamiltonian (1) to the following one

$$
\begin{aligned}
\widetilde{H}= & \frac{v_{1}^{2}+v_{2}^{2}}{8\left(u_{1}^{2}+u_{2}^{2}\right)}+\frac{1}{2}\left(u_{2} v_{1}-u_{1} v_{2}\right) \\
& -\varepsilon\left(u_{1}^{4}-4 u_{1}^{2} u_{2}^{2}+u_{2}^{4}\right)+\frac{\alpha^{3} \sigma}{u_{1}^{2}+u_{2}^{2}} .
\end{aligned}
$$

The corresponding Hamiltonian vector field is given by

$$
\begin{aligned}
\dot{u}_{1}= & \frac{v_{1}}{4\left(u_{1}^{2}+u_{2}^{2}\right)}+\frac{1}{2} u_{2}, \\
\dot{u}_{2}= & \frac{v_{2}}{4\left(u_{1}^{2}+u_{2}^{2}\right)}-\frac{1}{2} u_{1} \\
\dot{v}_{1}= & \frac{\left(v_{1}^{2}+v_{2}^{2}\right) u_{1}}{4\left(u_{1}^{2}+u_{2}^{2}\right)^{2}}+\frac{1}{2} v_{2}-\varepsilon\left(8 u_{1} u_{2}^{2}-4 u_{1}^{3}\right) \\
& +\frac{2 \alpha^{3} \sigma u_{1}}{\left(u_{1}^{2}+u_{2}^{2}\right)^{2}}, \\
\dot{v}_{2}= & \frac{\left(v_{1}^{2}+v_{2}^{2}\right) u_{2}}{4\left(u_{1}^{2}+u_{2}^{2}\right)^{2}}-\frac{1}{2} v_{1}-\varepsilon\left(8 u_{1}^{2} u_{2}-4 u_{2}^{3}\right) \\
& +\frac{2 \alpha^{3} \sigma u_{2}}{\left(u_{1}^{2}+u_{2}^{2}\right)^{2}} .
\end{aligned}
$$

We now perform the time change $t \rightarrow \tau$ such that $\frac{\mathrm{d} t}{\mathrm{~d} \tau}=4\left(u_{1}^{2}+u_{2}^{2}\right)$, which corresponds to multiplying this vector field by $4\left(u_{1}^{2}+u_{2}^{2}\right)$, and then restrict it to the 3-dimensional manifold given by $\widetilde{H}=-1 / 8$

$$
\begin{aligned}
& \left(v_{1}+2 u_{2}\left(u_{1}^{2}+u_{2}^{2}\right)\right) \frac{\partial}{\partial u_{1}}+\left(v_{2}-2 u_{1}\left(u_{1}^{2}+u_{2}^{2}\right)\right) \frac{\partial}{\partial u_{2}} \\
& +\left(\frac{u_{1} v_{1}^{2}+u_{1} v_{2}^{2}}{u_{1}^{2}+u_{2}^{2}}+2\left(u_{1}^{2}+u_{2}^{2}\right) v_{2}\right. \\
& +16 \varepsilon\left(u_{1}^{2}-2 u_{2}^{2}\right)\left(u_{1}^{2}+u_{2}^{2}\right) u_{1} \\
& \left.+\frac{8 \alpha^{3} \sigma u_{1}}{u_{1}^{2}+u_{2}^{2}}\right) \frac{\partial}{\partial v_{1}}+\left(\frac{u_{2} v_{1}^{2}+u_{2} v_{2}^{2}}{u_{1}^{2}+u_{2}^{2}}-2\left(u_{1}^{2}+2 u_{2}^{2}\right) v_{1}\right. \\
& \left.-16 \varepsilon\left(2 u_{1}^{2}-u_{2}^{2}\right)\left(u_{1}^{2}+u_{2}^{2}\right) u_{2}+\frac{8 \alpha^{3} \sigma u_{2}}{u_{1}^{2}+u_{2}^{2}}\right) \frac{\partial}{\partial v_{2}} \\
& =\left(v_{1}+2 u_{2}\left(u_{1}^{2}+u_{2}^{2}\right)\right) \frac{\partial}{\partial u_{1}}+\left(v_{2}-2 u_{1}\left(u_{1}^{2}+u_{2}^{2}\right)\right) \frac{\partial}{\partial u_{2}} \\
& +\left(-4 u_{1} u_{2} v_{1}+\left(6 u_{1}^{2}+2 u_{2}^{2}\right) v_{2}\right. \\
& +24 \varepsilon u_{1}\left(u_{1}^{4}-2 u_{1}^{2} u_{2}^{2}-u_{2}^{4}\right) \\
& \left.-u_{1}\right) \frac{\partial}{\partial v_{1}}+\left(\left(-2 u_{1}^{2}-6 u_{2}^{2}\right) v_{1}+4 u_{1} u_{2} v_{2}\right. \\
& \left.+24 \varepsilon u_{2}\left(u_{1}^{4}+2 u_{1}^{2} u_{2}^{2}-u_{2}^{4}\right)-u_{2}\right) \frac{\partial}{\partial v_{2}} \\
& +8\left(\widetilde{H}+\frac{1}{8}\right)\left(u_{1} \frac{\partial}{\partial v_{1}}+u_{2} \frac{\partial}{\partial v_{2}}\right) .
\end{aligned}
$$

It now appears that on $\widetilde{H}=-1 / 8$, this last representation does not contain the parameter $\alpha$ nor any singularity. More- 
over, this vector field is Hamiltonian on $\mathbb{C}^{4}$ with Hamiltonian

$$
\begin{aligned}
\widehat{H}= & \frac{1}{2}\left(v_{1}^{2}+v_{2}^{2}\right)+2\left(u_{2} v_{1}-u_{1} v_{2}\right)\left(u_{1}^{2}+u_{2}^{2}\right) \\
& -4 \varepsilon\left(u_{1}^{2}+u_{2}^{2}\right)\left(u_{1}^{4}-4 u_{1}^{2} u_{2}^{2}+u_{2}^{4}\right) \\
& +\frac{1}{2}\left(u_{1}^{2}+u_{2}^{2}\right) .
\end{aligned}
$$

The relation between first integrals of original (9) and regularised (13) Hamiltonian is described by the following proposition.

Proposition 1 For $\sigma \neq 0$, if Hamiltonian (9) admits an additional first integral rational in $x, y, p_{x}, p_{y}, r$, then Hamiltonian (13) admits an additional rational first integral in $u_{1}, u_{2}, v_{1}, v_{2}$.

Proof Assume there exists a rational first integral $I$ in $x, y, p_{x}, p_{y}, r$ of (9) that is functionally independent of $H$. Then, performing the coordinates change (10) on $I$ gives $\tilde{I}$ which is a rational expression in $u_{1}, u_{2}, v_{1}, v_{2}, \alpha$, still independent with $\widetilde{H}$. The function $\widetilde{I}$ is still a first integral of the vector field after multiplying it by $4\left(u_{1}^{2}+u_{2}^{2}\right)$. Thus, vector field (12) in dimension 5 (with additional variable $\alpha$ ) admits 3 first integrals $\widetilde{H}, \widetilde{I},-4 \alpha^{3} \sigma$, functionally independent as $\sigma \neq 0$.

We consider the invariant surface $\Sigma=\{\widetilde{H}=-1 / 8\}$, which is the zero level of the polynomial $\widehat{H}+4 \alpha^{3} \sigma \in$ $\mathbb{C}[u, v, \alpha]$, where $u=\left(u_{1}, u_{2}\right)$ and $v=\left(v_{1}, v_{2}\right)$. Let us check that this polynomial is irreducible. This polynomial is of degree 2 in $v$. If it factors with a factor of degree 0 in $v$, then $\widehat{H}+4 \alpha^{3} \sigma$ should have a factor in $u$ only, which is impossible because the term $\frac{1}{2}\left(v_{1}^{2}+v_{2}^{2}\right)$ always appears. Thus, it should factorise in two terms of degree 1 in $v$

$$
\widehat{H}+4 \alpha^{3} \sigma=\frac{1}{2}\left(a_{1} v_{1}+a_{2} v_{2}+a_{3}\right)\left(b_{1} v_{1}+b_{2} v_{2}+b_{3}\right)
$$

with $a_{i}, b_{i}$ polynomials in $u$. Expanding the right-hand side, we find that up to a constant factor $a_{1}=1, b_{1}=1, a_{2}=$ $\mathrm{i}, b_{2}=-\mathrm{i}$ and then

$$
\begin{aligned}
a_{3}+b_{3} & =4 u_{2}\left(u_{1}^{2}+u_{2}^{2}\right),-\mathrm{i} a_{3}+\mathrm{i} b_{3} \\
& =-4 u_{1}\left(u_{1}^{2}+u_{2}^{2}\right)
\end{aligned}
$$

and thus the only possible factorisation would be

$$
\begin{aligned}
& \frac{1}{2}\left(v_{1}+\mathrm{i} v_{2}+2\left(u_{2}-\mathrm{i} u_{1}\right)\left(u_{1}^{2}+u_{2}^{2}\right)\right) \\
& \left(v_{1}-\mathrm{i} v_{2}+2\left(u_{2}+\mathrm{i} u_{1}\right)\left(u_{1}^{2}+u_{2}^{2}\right)\right) \\
& =\frac{1}{2}\left(v_{1}^{2}+v_{2}^{2}\right)+2\left(u_{2} v_{1}-u_{1} v_{2}\right)\left(u_{1}^{2}+u_{2}^{2}\right) \\
& +2\left(u_{1}^{2}+u_{2}^{2}\right)^{3},
\end{aligned}
$$

which is never equal to $\widehat{H}+4 \alpha^{3} \sigma$. Thus, this polynomial is irreducible.

We can now restrict the vector field (12) to $\Sigma$ which gives a Hamiltonian vector field with Hamiltonian $\widehat{H}$. We notice that $-4 \alpha^{3} \sigma$ restricted on $\Sigma$ equals $\widehat{H}$. Thanks to Ziglin Lemma [23,24], up to replacing $\widetilde{I}$ by an algebraic expression in $\widetilde{I},-4 \alpha^{3} \sigma$, we can ensure that the restriction of $\widetilde{I}$ to $\Sigma$ is a well-defined function $\widehat{I}$ which is functionally independent with $\widehat{H}$. Thus, $\widehat{I}$ is a functionally independent first integral of the Hamiltonian vector field of $\widehat{H}$.

Remark that when $\sigma=0$, the hypersurface $\Sigma$ is defined by $\widehat{H}=0$. Restricting the vector field on $\Sigma$, we obtain equations corresponding to the Hamiltonian vector field of $\widehat{H}$ and equation $\frac{\mathrm{d} \alpha}{\mathrm{d} \tau}=0$. So the restriction of $\widetilde{I}$ to $\Sigma$ still gives a first integral $\widehat{I}$ which, after fixing $\alpha$ to an arbitrary constant, will give a first integral of $\widehat{H}$ on its zero level. As the initial Hamiltonian $H$ is integrable for $\sigma=0$, this implies that Hamiltonian $\widehat{H}$ is integrable on its zero level.

This property explains the further observation why the variational equations for a particular solution lying on the zero level of $\widehat{H}$ have Liouvillian solutions, that is their differential Galois group is solvable. Indeed, first-order variational equations restricted to the zero energy level will have a virtually Abelian Galois group, and in the supplementary space, normal to the surface $\widehat{H}=0$, the variational equations can then be solved by variation of constants method.

Following [18] to simplify search of invariant manifolds and particular solutions lying on them we make the next canonical change of variables

$$
\left[\begin{array}{l}
\boldsymbol{u} \\
\boldsymbol{v}
\end{array}\right]=\left[\begin{array}{cc}
\boldsymbol{A} & 0 \\
0 & \left(\boldsymbol{A}^{-1}\right)^{T}
\end{array}\right]\left[\begin{array}{l}
\boldsymbol{x} \\
\boldsymbol{y}
\end{array}\right], \quad \boldsymbol{A}=\frac{1}{\sqrt{2}}\left[\begin{array}{ll}
1 & \mathrm{i} \\
\mathrm{i} & 1
\end{array}\right],
$$

where $\boldsymbol{x}=\left[x_{1}, x_{2}\right]^{T}$ and $\boldsymbol{y}=\left[y_{1}, y_{2}\right]^{T}$. After this transformation Hamiltonian (13) takes the form

$$
\begin{aligned}
H= & \mathrm{i}\left(x_{1} x_{2}-y_{1} y_{2}\right)-4 x_{1} x_{2}\left(x_{1} y_{1}-x_{2} y_{2}\right) \\
& -4 \mathrm{i} \varepsilon x_{1} x_{2}\left(3 x_{1}^{4}-2 x_{1}^{2} x_{2}^{2}+3 x_{2}^{4}\right)
\end{aligned}
$$

and Hamilton equations are

$$
\begin{aligned}
x_{1}^{\prime}= & -\mathrm{i} y_{2}-4 x_{1}^{2} x_{2}, \\
x_{2}^{\prime}= & -\mathrm{i} y_{1}+4 x_{1} x_{2}^{2}, \\
y_{1}^{\prime}= & x_{2}\left[8 x_{1} y_{1}-4 x_{2} y_{2}\right. \\
& \left.+12 \mathrm{i} \varepsilon\left(5 x_{1}^{4}-2 x_{1}^{2} x_{2}^{2}+x_{2}^{4}\right)-\mathrm{i}\right], \\
y_{2}^{\prime}= & x_{1}\left[4 x_{1} y_{1}-8 x_{2} y_{2}\right. \\
& \left.+12 \mathrm{i} \varepsilon\left(x_{1}^{4}-2 x_{1}^{2} x_{2}^{2}+5 x_{2}^{4}\right)-\mathrm{i}\right],
\end{aligned}
$$

where ' denotes differentiation with respect to $\tau$. In these variables one can easily notice that two planes

$$
\begin{aligned}
& \mathcal{M}_{1}=\left\{\left(x_{1}, x_{2}, y_{1}, y_{2}\right) \in \mathbb{C}^{4} \mid x_{2}=y_{1}=0\right\}, \\
& \mathcal{M}_{2}=\left\{\left(x_{1}, x_{2}, y_{1}, y_{2}\right) \in \mathbb{C}^{4} \mid x_{1}=y_{2}=0\right\}
\end{aligned}
$$

are invariant.

\section{Integrability analysis}

The system restricted to invariant plane $\mathcal{M}_{1}$ reads

$$
x_{1}^{\prime}=-\mathrm{i} y_{2}, \quad y_{2}^{\prime}=\mathrm{i} x_{1}\left(12 \varepsilon x_{1}^{4}-1\right) .
$$


It has first integral

$h=-\frac{1}{2} y_{2}^{2}+\frac{1}{2} x_{1}^{2}-2 \varepsilon x_{1}^{6}$.

Thus, we have to our disposal a family of particular solutions parametrised by value of $h$. Let $\left[X_{1}, X_{2}, Y_{1}, Y_{2}\right]^{T}$ denote variations of variables $\left[x_{1}\right.$, $\left.x_{1}, y_{1}, y_{2}\right]^{T}$. Then, variational equations along a chosen particular solution take the form

$$
\left[\begin{array}{c}
X_{1}^{\prime} \\
Y_{2}^{\prime} \\
X_{2}^{\prime} \\
Y_{1}^{\prime}
\end{array}\right]=\left[\begin{array}{cccc}
0 & -\mathrm{i} & -4 x_{1}^{2} & 0 \\
\mathrm{i}\left(60 \varepsilon x_{1}^{4}-1\right) & 0 & -8 x_{1} y_{2} & 4 x_{1}^{2} \\
0 & 0 & 0 & -\mathrm{i} \\
0 & 0 & \mathrm{i}\left(60 \varepsilon x_{1}^{4}-1\right) & 0
\end{array}\right]\left[\begin{array}{c}
X_{1} \\
Y_{2} \\
X_{2} \\
Y_{1}
\end{array}\right] .
$$

For further analysis we need a proper choice of a particular solution. Let us fix $h=e$. Then, from (18) we get a hyperelliptic curve

$y_{2}^{2}=P_{6}\left(x_{1}\right), \quad P_{6}\left(x_{1}\right)=-2 e+x_{1}^{2}-4 \varepsilon x_{1}^{6}$.

For a generic value of $e$ the particular solution corresponding to this curve is given by elliptic functions and their integrals. We simplify considerably our further analysis by taking $e$ such that $108 e^{2} \varepsilon=1$. For this choice the discriminant of polynomial $P_{6}\left(x_{1}\right)$ vanishes, so the hyper-elliptic curve (19) degenerates and, in effect, particular solutions can be expressed by elementary functions. Then, we change independent variable $\tau \rightarrow z=\frac{1}{3 e} x_{1}^{2}(\tau)$. Variational equations transform into

$$
\frac{\mathrm{d}}{\mathrm{d} z}\left[\begin{array}{c}
X_{1} \\
Y_{2} \\
X_{2} \\
Y_{1}
\end{array}\right]=\frac{1}{r(z)}\left[\begin{array}{cccc}
0 & -\mathrm{i} & -12 e z & 0 \\
\mathrm{i}\left(5 z^{2}-1\right) & 0 & -12 \mathrm{i} e r(z) & 12 e z \\
0 & 0 & 0 & \mathrm{i} \\
0 & 0 & \mathrm{i}\left(5 z^{2}-1\right) & 0
\end{array}\right]\left[\begin{array}{c}
X_{1} \\
Y_{2} \\
X_{2} \\
Y_{1}
\end{array}\right],
$$

where

$r(z)=\frac{2}{\sqrt{3}}(z-1) \sqrt{z(z+2)}$.

We make the next change of independent variable $z \rightarrow u$ defined as $z=\frac{(u+1)^{2}}{2(u+2)}$ that removes square roots from coefficients of variational equations (20). Moreover, we rescale dependent variables

$\left[\widetilde{X}_{1}, \widetilde{Y}_{2}, \widetilde{X}_{2}, \widetilde{Y}_{1}\right]^{T}=\operatorname{diag}(-\mathrm{i}, \sqrt{3},-6 \mathrm{i} \sqrt{3} e, 18 e)\left[X_{1}, Y_{2}, X_{2}, Y_{1}\right]^{T}$.

Transformed variational equations take the form

$\frac{\mathrm{d}}{\mathrm{d} u}\left[\begin{array}{c}\widetilde{X}_{1} \\ \widetilde{Y}_{2} \\ \widetilde{X}_{2} \\ \widetilde{Y}_{1}\end{array}\right]=\left[\begin{array}{cccc}0 & \frac{1}{u^{2}-3} & \frac{(u+1)^{2}}{(u+2)\left(u^{2}-3\right)} & 0 \\ p(u) & 0 & \frac{(u+1)(u+3)}{(u+2)^{2}} & -\frac{(u+1)^{2}}{(u+2)\left(u^{2}-3\right)} \\ 0 & 0 & 0 & \frac{1}{u^{2}-3} \\ 0 & 0 & p(u) & 0\end{array}\right]\left[\begin{array}{c}\widetilde{X}_{1} \\ \widetilde{Y}_{2} \\ \widetilde{X}_{2} \\ \widetilde{Y}_{1},\end{array}\right]$,

$p(u)=\frac{3\left(-11+4 u+26 u^{2}+20 u^{3}+5 u^{4}\right)}{4(u+2)^{2}\left(u^{2}-3\right)}$.
This system gives the fourth-order equation for variable $\widetilde{X}_{1}$ of the form

$\frac{\mathrm{d}^{4} \widetilde{X}_{1}}{\mathrm{~d} u^{4}}+a_{3} \frac{\mathrm{d}^{3} \widetilde{X}_{1}}{\mathrm{~d} u^{3}}+a_{2} \frac{\mathrm{d}^{2} \tilde{X}_{1}}{\mathrm{~d} u^{2}}+a_{1} \frac{\mathrm{d} \widetilde{X}_{1}}{\mathrm{~d} u}+a_{0} \widetilde{X}_{1}=0$,

with coefficients

$$
\begin{aligned}
a_{3}= & \frac{4\left(3+12 u+21 u^{2}+12 u^{3}+2 u^{4}\right)}{(u+1)(u+2)(3+u)\left(-3+u^{2}\right)}, \\
a_{2}= & \frac{177+972 u+1755 u^{2}+1560 u^{3}+711 u^{4}+156 u^{5}+13 u^{6}}{2(u+1)^{2}(u+2)^{2}(u+3)^{2}\left(u^{2}-3\right)}, \\
a_{1}= & -\frac{108+885 u+1980 u^{2}+2301 u^{3}+1572 u^{4}+627 u^{5}+132 u^{6}+11 u^{7}}{(u+1)^{2}(u+2)^{2}(u+3)^{2}\left(u^{2}-3\right)^{2}}, \\
a_{0}= & \frac{3}{16(u+1)^{2}(u+2)^{4}(u+3)^{2}\left(u^{2}-3\right)^{3}} \\
& {\left[-129+32640 u+131115 u^{2}+238992 u^{3}+262146 u^{4}\right.} \\
& \left.+192336 u^{5}+98570 u^{6}+35312 u^{7}+8447 u^{8}+1200 u^{9}+75 u^{10}\right] .
\end{aligned}
$$

This equation has the following general solution

$\widetilde{X}_{1}(u)=C_{1} v_{1}(u)+C_{2} v_{2}(u)+C_{3} v_{3}(u)+C_{4} v_{4}(u)$,

with fundamental solutions

$$
\begin{aligned}
& v_{1}=\frac{(3+u)\left(u^{2}-3\right)}{(2+u)^{3 / 2}}, \\
& v_{2}=-\frac{5 \sqrt{3}(3+u)\left(u^{2}-3\right) \operatorname{arctanh}\left(\frac{u}{\sqrt{3}}\right)}{432(2+u)^{3 / 2}} \\
& +\frac{5 u^{4}+15 u^{3}-25 u^{2}-111 u-84}{(2+u)^{3 / 2}\left(144 u^{2}-432\right)}, \\
& v_{3}=-\frac{25 \sqrt{3}(3+u)\left(u^{2}-3\right) \operatorname{arctanh}\left(\frac{u}{\sqrt{3}}\right)}{96(2+u)^{3 / 2}} \\
& +\frac{(3+u)\left(u^{2}-3\right) \ln (2+u)}{4(2+u)^{3 / 2}}+\frac{9 u^{4}+75 u^{3}-29 u^{2}-555 u-564}{(2+u)^{3 / 2}\left(32 u^{2}-96\right)}, \\
& v_{4}=-\frac{5(3+u)\left(u^{2}-3\right) \sqrt{3}}{(2+u)^{3 / 2}} \int \frac{\operatorname{arctanh}(u / \sqrt{3})}{2+u} \mathrm{~d} u \\
& +\left[\frac{5 \sqrt{3}(3+u)\left(u^{2}-3\right)}{2(2+u)^{3 / 2}} \ln (2+u)\right. \\
& \left.-\frac{5\left(u^{2}-3\right)(108+11 u \sqrt{3}-39 \sqrt{3}+36 u)}{72(2+u)^{3 / 2}}\right] \operatorname{arctanh}\left(\frac{u}{\sqrt{3}}\right) \\
& +\frac{\left(-12 u^{5}-51 u^{4}+27 u^{3}+291 u^{2}+225 u-72\right)}{(2+u)^{3 / 2}\left(2 u^{2}-6\right)} \ln (u+2) \\
& -\frac{5(3+u)\left(u^{2}-3\right)}{2(2+u)^{3 / 2}} \ln (u-\sqrt{3})+\frac{(51+17 u)\left(u^{2}-3\right)}{4(2+u)^{3 / 2}} \ln \left(u^{2}-3\right) \\
& +\frac{-17 u^{4}-123 u^{3}+805 u^{2}+3387 u+2964}{(2+u)^{3 / 2}\left(24 u^{2}-72\right)} \text {. }
\end{aligned}
$$

Let us consider integral which appears in the first term of $v_{4}$. Function $\operatorname{arctanh}(x)$ is a multi-valued function so we have to consider it on the corresponding Riemann surface, see Fig. 10. Values of $\operatorname{arctanh}(x)$ on different sheets of the 


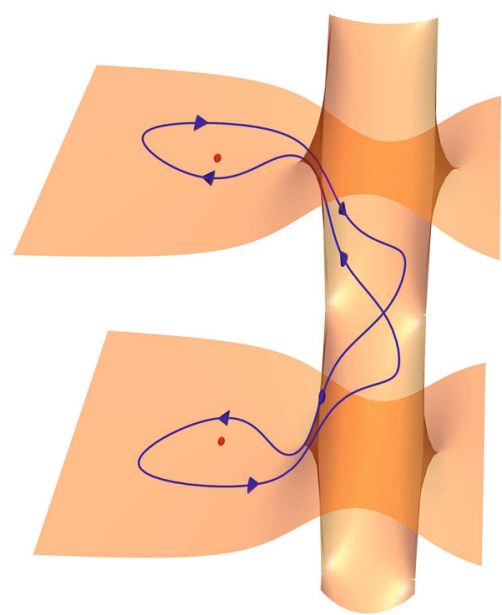

Fig. 10 Riemann surface of $\operatorname{arctanh}(x)$ with the commutator path

Riemann surface differ by integer multiples of $i \pi$. Thus, we consider integral

$$
\begin{aligned}
I(\alpha) & =\int \frac{1}{2+u}\left[\operatorname{arctanh}\left(\frac{u}{\sqrt{3}}\right)+\alpha\right] \mathrm{d} u \\
& =r(\alpha) \ln (u+2)+\sum_{n \geq 0} a_{n}(u+2)^{n},
\end{aligned}
$$

where using the principal value of arctanh we have

$r(\alpha)=\alpha+\operatorname{arctanh}\left(\frac{-2}{\sqrt{3}}\right)=\alpha+\ln (2-\sqrt{3})+\mathrm{i} \frac{\pi}{2}$.

The form of the right-hand side of $I(\alpha)$ follows from the fact that $\operatorname{arctanh}(u / \sqrt{3})$ is smooth at $u=-2$.

We investigate the monodromy group of this integral. To this end we take two loops $\sigma$ and $\gamma$ with one common point $u_{0}$. They are ovals encircling counter-clockwise points $u=$ -2 , and $u=-\sqrt{3}$, respectively, see Fig. 11 .

Let $M_{\sigma}$ and $M_{\gamma}$ denote the monodromy operators corresponding to the respective loops. Then,

$M_{\sigma}(I(\alpha))=I(\alpha)+2 \pi \mathrm{i} r(\alpha)$,

and

$M_{\gamma}\left(\operatorname{arctanh}\left(\frac{u}{\sqrt{3}}\right)+\alpha\right)=\operatorname{arctanh}\left(\frac{u}{\sqrt{3}}\right)+\alpha+\pi \mathrm{i}$,

so

$M_{\gamma}(I(\alpha))=I(\alpha+\pi \mathrm{i})$.

In effect, for the commutator $M=M_{\gamma}^{-1} M_{\sigma}^{-1} M_{\gamma} M_{\sigma}$ we obtain

$$
\begin{aligned}
M(I(\alpha)) & =I(\alpha)+2 \mathrm{i} \pi r(\alpha)-2 \mathrm{i} \pi r(\alpha+\mathrm{i} \pi) \\
& =I(\alpha)+2 \pi^{2},
\end{aligned}
$$

thus the monodromy group is not commutative. But this group is a subgroup of the differential Galois group $\mathcal{G}(L / \mathbb{C}(u))$ of the extension

$$
\mathbb{C}(u) \subset L=\mathbb{C}\left(u, \operatorname{arctanh}\left(\frac{u}{\sqrt{3}}\right), I(\alpha)\right) .
$$

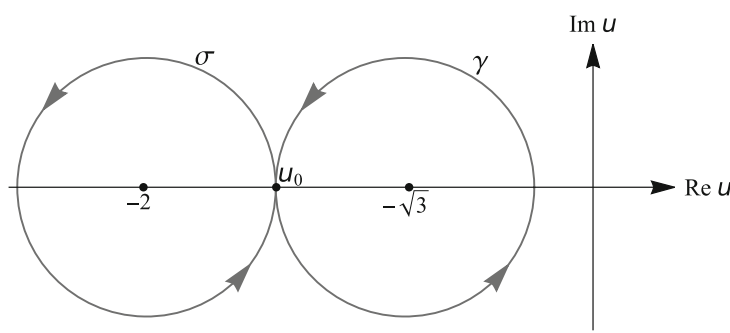

(a) loops around singularities

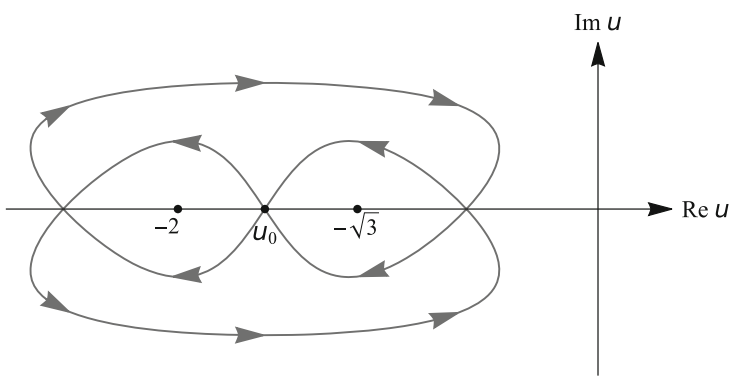

(b) commutator contour

Fig. 11 Contours for monodromy calculations

In fact, $\mathcal{G}(L / \mathbb{C}(u))$ is the Zariski closure of the monodromy group and it is connected because the extension $\mathbb{C}(u) \subset L$ was obtained by two successive quadratures. The field $L$ is a subfield of the Picard-Vessiot field of the variational equations and thus its identity component is not Abelian. This fact shows that the generalised Hill system is not integrable.

The differential Galois methods used in our proof of Theorem 1 are algebraic and they do not characterise the dynamical origin of the non-integrability. So, in general, there is no direct connections between chaotic behaviour of the system and the non-integrability demonstrated with the help of differential Galois methods. However, there are certain exceptions. For example, in article [25] it was shown how the necessary conditions for splitting of the separatrices can be obtained with the help of differential Galois techniques.

\section{Super-integrability of particular cases}

\subsection{The parametric Hénon case}

We mentioned about the integrability of the parametric Hénon system

$$
\begin{aligned}
\mathscr{H}= & \frac{1}{2}\left(p_{x}^{2}+p_{y}^{2}\right)+y p_{x}-x p_{y} \\
& +\varepsilon\left(-x^{2}+\frac{1}{2} y^{2}\right) \\
\dot{x}= & p_{x}+y, \quad \dot{p}_{x}=p_{y}+2 \varepsilon x, \\
\dot{y}= & p_{y}-x, \quad \dot{p}_{y}=-p_{x}-\varepsilon y,
\end{aligned}
$$

for all values of $\varepsilon$. Additional first integral was given in (2). 
But for certain values of $\varepsilon$ this system appears to be superintegrable. In order to understand origin of these additional first integrals and values of $\varepsilon$ for which this system is superintegrable, we will study first integrals of the Hamilton equations (26) which are in fact linear

$\frac{\mathrm{d} \boldsymbol{x}}{\mathrm{d} t}=\mathbb{A} \boldsymbol{x}$

with matrix

$\mathbb{A}=\left[\begin{array}{cccc}0 & 1 & 1 & 0 \\ -1 & 0 & 0 & 1 \\ 2 \varepsilon & 0 & 0 & 1 \\ 0 & -\varepsilon & -1 & 0\end{array}\right]$

and $\boldsymbol{x}=\left[x, y, p_{x}, p_{y}\right]^{T}$. The characteristic polynomial of this matrix is

$$
\begin{aligned}
p(\lambda) & =\operatorname{det}[\mathbb{A}-\lambda \mathbb{I}] \\
& =\lambda^{4}+(2-\varepsilon) \lambda^{2}+\varepsilon-2 \varepsilon^{2}+1 .
\end{aligned}
$$

Substituting $\lambda=\mathrm{i} \omega$, where $\omega \in \mathbb{R}$, the characteristic equation takes the form

$\omega^{4}+(\varepsilon-2) \omega^{2}+\varepsilon-2 \varepsilon^{2}+1=0$.

Solving this equation in $\omega$ gives up to sign two solutions $\pm \omega_{1}, \pm \omega_{2}$ with

$$
\begin{aligned}
& \omega_{1}=\frac{1}{\sqrt{2}} \sqrt{2-\varepsilon-\sqrt{\varepsilon(9 \varepsilon-8)}}, \\
& \omega_{2}=\frac{1}{\sqrt{2}} \sqrt{2-\varepsilon+\sqrt{\varepsilon(9 \varepsilon-8)}} .
\end{aligned}
$$

These solutions are distinct when $\varepsilon(9 \varepsilon-8) \neq 0$ and $\omega_{1} \omega_{2} \neq 0$. When matrix $\mathbb{A}$ is diagonalisable, system (27) after a basis change is diagonal and its solutions are $\exp \left( \pm \mathrm{i} \omega_{1} t\right), \exp \left( \pm \mathrm{i} \omega_{2} t\right)$. System (27) admits 3 independent first integrals if and only if the transcendence degree of the field generated by the solutions is $4-3=1$. Thus, for super-integrability, $\exp \left( \pm \mathrm{i} \omega_{1} t\right), \exp \left( \pm \mathrm{i} \omega_{2} t\right)$ should be algebraically dependant, and so $\omega_{1} / \omega_{2} \in \mathbb{Q}$.

When $\varepsilon=0,8 / 9$, matrix $\mathbb{A}$ is not diagonalisable, and the solution field is, respectively, $\mathbb{C}(t, \exp (\mathrm{i} t))$ and $\mathbb{C}(t, \exp (\mathrm{i} \sqrt{5} t / 3))$. Thus, the transcendence degree is 2 , and the system is not super-integrable. Stays the case $\omega_{1} \omega_{2}=-16(2 \varepsilon+1)(\varepsilon-1)=0$ which gives $\varepsilon=$ $1,-1 / 2$. These cases give solutions fields $\mathbb{C}(t, \exp (\mathrm{i} t))$ and $\mathbb{C}(t$, $\exp (\mathrm{i} t \sqrt{10} / 2))$, respectively, and so are not superintegrable.

Now we can explain the existence of an additional first integral and super-integrability for selected values of $\varepsilon$. Let us define function

$\kappa(\varepsilon):=\frac{\omega_{1}}{\omega_{2}}$.

As $\omega_{1}, \omega_{2}$ are defined up to sign and permutation, we can assume $\kappa \in[0,1]$, and this allows to restrict the study of $\kappa(\varepsilon)$ to $\varepsilon \in\left(-\frac{1}{2}, 0\right) \cup\left(\frac{8}{9}, 1\right)$. It is continuous and monotone on the two intervals, see Fig. 12. by

Inverse of this function in interval $\varepsilon \in\left(-\frac{1}{2}, 0\right)$ is given

$$
\varepsilon_{-}(\kappa)=\frac{\kappa^{4}+6 \kappa^{2}+1-\left(\kappa^{2}+1\right) \sqrt{9 \kappa^{4}-2 \kappa^{2}+9}}{2\left(2 \kappa^{4}+5 \kappa^{2}+2\right)},
$$

while in interval $\varepsilon \in\left(\frac{8}{9}, 1\right)$ it reads

$\varepsilon_{+}(\kappa)=\frac{\kappa^{4}+6 \kappa^{2}+1+\left(\kappa^{2}+1\right) \sqrt{9 \kappa^{4}-2 \kappa^{2}+9}}{2\left(2 \kappa^{4}+5 \kappa^{2}+2\right)}$,

see Fig 13.

System (25) is super-integrable if and only if $\kappa \in(0,1) \cap$ $\mathbb{Q}$, and thus for $\varepsilon$ in

$\left\{\varepsilon_{-}(\kappa), \kappa \in(0,1) \cap \mathbb{Q}\right\} \cup\left\{\varepsilon_{+}(\kappa), \kappa \in(0,1) \cap \mathbb{Q}\right\}$.

Now the first integrals can be obtained by diagonalising $\mathbb{A}$, and when $\kappa \in \mathbb{Q}$, and the multiplicative relations between the exponentials give 3 independent first integrals. Two of them come from the relations $e^{\mathrm{i} \omega_{1} t} e^{-\mathrm{i} \omega_{1} t}=$ $1, \quad e^{i \omega_{2} t} e^{-i \omega_{2} t}=1$ and the exceptional additional one from $\left(e^{\mathrm{i} \omega_{1} t}\right)^{m_{2}}\left(e^{-\mathrm{i} \omega_{2} t}\right)^{m_{1}}=1, \quad \kappa=\frac{m_{1}}{m_{2}}$,

where $m_{1}, m_{2} \in \mathbb{Z}$. This will give a polynomial first integral of degree $\left|m_{1}\right|+\left|m_{2}\right|$ in momenta for $\kappa \in(0,1) \cap \mathbb{Q}$.

For example for $\kappa=1 / 2, \varepsilon=\frac{1}{108}(41 \pm 5 \sqrt{145})$ this system has an additional first integral of degree three in momenta

$$
\begin{aligned}
G= & 62(-25+\sqrt{145}) p_{x}^{3}+27(5-13 \sqrt{145}) p_{x}^{2} y \\
& +5\left(972 p_{y}^{2}+9(139+13 \sqrt{145}) p_{y} x\right. \\
& \left.+(752+68 \sqrt{145}) x^{2}\right) y \\
& -9 p_{x}\left(15\left(p_{y}-x\right)\left(36 p_{y}+(19+\sqrt{145}) x\right)\right. \\
& \left.+(155+11 \sqrt{145}) y^{2}\right)-6(65+2 \sqrt{145}) y^{3},
\end{aligned}
$$

and for $\kappa=1 / 3, \varepsilon=\frac{68}{209} \pm \frac{60}{209} \sqrt{5}$ of degree four in momenta

$$
\begin{aligned}
G= & 10658164 p_{y}^{4}+2402455(83 \mp 29 \sqrt{5}) p_{y}^{3} x \\
& -43681(6733 \mp 3011 \sqrt{5}) p_{x}^{3} y \\
& -209 p_{y} x\left(209(-6733 \pm 3011 \sqrt{5}) p_{x}^{2}\right. \\
& +88(-7506 \pm 2297 \sqrt{5}) x^{2} \\
& +10032(-131 \pm 59 \sqrt{5}) p_{x} y \\
& \left.+8(25532 \mp 11047 \sqrt{5}) y^{2}\right) \\
& +8\left(4598(-343 \pm 283 \sqrt{5}) x^{4}\right. \\
& +y^{3}\left(209(-32444 \pm 14513 \sqrt{5}) p_{x}\right. \\
& +4(821453 \mp 367275 \sqrt{5}) y) \\
& +209 x^{2} y\left(11(-10250 \pm 4561 \sqrt{5}) p_{x}\right. \\
& +6(4157 \mp 1889 \sqrt{5}) y)) \\
& +209 p_{y}^{2}\left(5016(-201 \pm 118 \sqrt{5}) x^{2}\right. \\
& +y\left(209(-3589 \pm 1595 \sqrt{5}) p_{x}\right. \\
& +1464(-47 \pm 20 \sqrt{5}) y)) .
\end{aligned}
$$


Fig. 12 Function $\kappa(\varepsilon)$

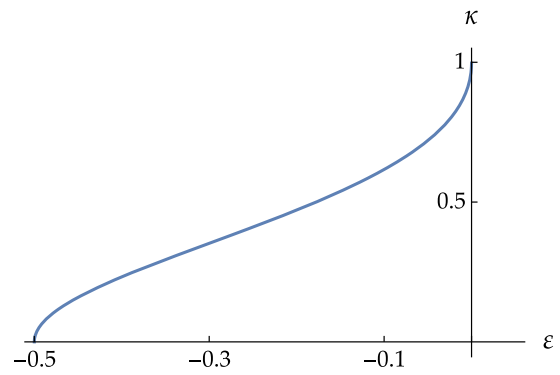

(a) $\varepsilon \in\left(-\frac{1}{2}, 0\right)$

Fig. 13 Inverse function $\varepsilon(\kappa)$

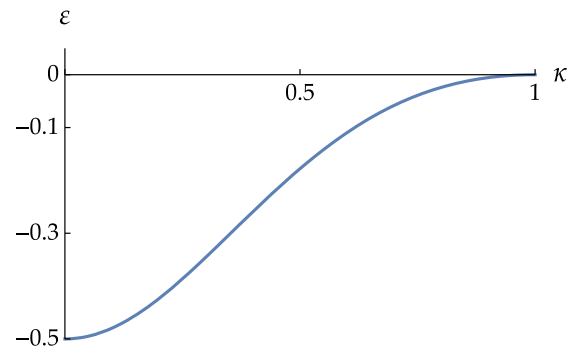

(a) $\varepsilon \in\left[-\frac{1}{2}, 0\right]$

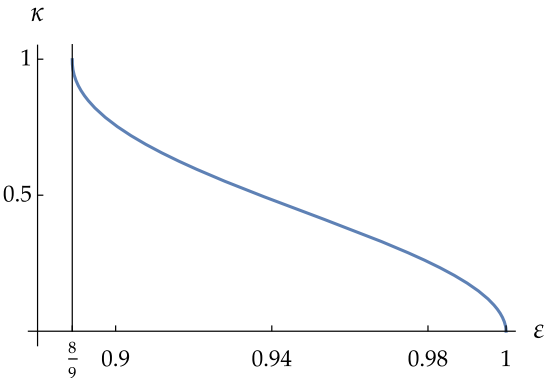

(b) $\varepsilon \in\left(\frac{8}{9}, 1\right)$

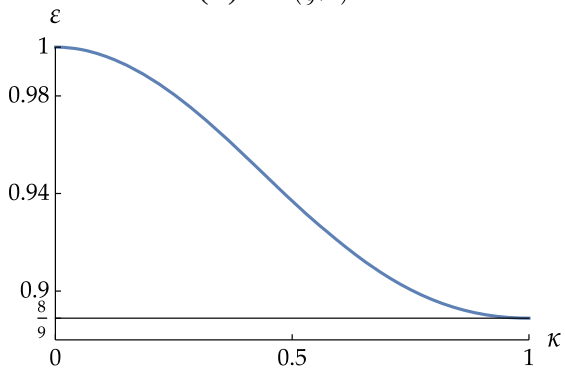

(b) $\varepsilon \in\left[\frac{8}{9}, 1\right]$

\subsection{The synodical Kepler case}

When $\varepsilon=0, \sigma \neq 0$ in (1), the Hamiltonian

$\mathscr{H}=\frac{1}{2}\left(p_{x}^{2}+p_{y}^{2}\right)+y p_{x}-x p_{y}+\frac{\sigma}{|\boldsymbol{r}|}$

describes a Keplerian motion in a rotating frame. The system admits an additional first integral, the angular momentum $p_{x} y-p_{y} x$, and we wonder if there could exist an independent third first integral. Denoting $\delta=\operatorname{sign}(\sigma)$, a hyperbolic orbit will satisfy in polar coordinates

$r(t)=\frac{p}{e \cos (\theta(t)+t)-\delta}, \quad p=a\left(e^{2}-1\right)$,

with eccentricity $e \neq 0$ and semi-major axis $a>0$. Let us introduce the eccentric anomaly angle $E(t)$

$\tanh \left(\frac{E(t)}{2}\right)=\sqrt{\frac{\delta e+1}{\delta e-1}} \tan \left(\frac{\theta(t)}{2}\right)$.

The eccentric anomaly satisfies Kepler's equation

$\tau t=e \sinh E(t)+\delta E(t)$,

for some $\tau$ depending on the orbit chosen and value of parameter $\sigma$.

Let us now assume that a third rational first integral exists. Then, the orbit is algebraic, and so there exists an algebraic relation between $r(t)$ and $e^{i \theta(t)}$, and thus

$f_{1}\left(e^{\mathrm{i} \theta(t)}\right)(e \cos (\theta(t)+t)-\delta)=p$

for an algebraic function $f_{1}$. Expanding the cosine and expressing it in function of $e^{\mathrm{i} \theta(t)}, e^{\mathrm{i} t}$, we then have an algebraic relation $e^{\mathrm{i} t}=f_{2}\left(e^{\mathrm{i} \theta(t)}\right)$. Now using the equation for the eccentric anomaly $E(t)$, we have that $e^{\mathrm{i} \theta(t)}$ can be expressed algebraically in terms of $e^{E(t)}$, and thus $e^{\mathrm{i} t}=f_{3}\left(e^{E(t)}\right)$, for an algebraic function $f_{3}$. Now taking the exponential of the Kepler equation (33), we have $\left(e^{-\mathrm{i} t}\right)^{\alpha}=e^{\delta E(t)} e^{e \sinh E(t)}$, where $\alpha=-\mathrm{i} \tau$. Denoting $z(t)=\exp (E(t))$, this relation writes

$f_{3}(z)=z^{\frac{\delta}{\alpha}} e^{\frac{e}{2 \alpha}\left(z-z^{-1}\right)}$,

on a certain non-empty open set of $z \in \mathbb{C}$. As $e \neq 0$, the right-hand side is a transcendental function of $z$, and the left-hand side should be an algebraic function. Thus, a third rational first integral cannot exist.

Acknowledgements For TC this research has been supported by a CNRS IEA Grant "Integrability of Hamiltonian Systems" and the work of AJM and MP was partially funded by The National Science Center of Poland under Grant No. 2020/39/D/ST1/01632. For the purpose of Open Access, the author has applied a CC-BY public copyright license to any Author Accepted Manuscript (AAM) version arising from this submission.

Data availability Data sharing is not applicable to this article as no new data were created or analysed in this study.

\section{Declarations}

Conflict of interest The authors declare that they have no conflict of interest.

Open Access This article is licensed under a Creative Commons Attribution 4.0 International License, which permits use, 
sharing, adaptation, distribution and reproduction in any medium or format, as long as you give appropriate credit to the original author(s) and the source, provide a link to the Creative Commons licence, and indicate if changes were made. The images or other third party material in this article are included in the article's Creative Commons licence, unless indicated otherwise in a credit line to the material. If material is not included in the article's Creative Commons licence and your intended use is not permitted by statutory regulation or exceeds the permitted use, you will need to obtain permission directly from the copyright holder. To view a copy of this licence, visit http://creativecommons.org/licenses/ by/4.0/.

\section{References}

1. Szebehely, V.: Theory of Orbit: The Restricted Three Body Problem. Academic Press (1967)

2. Hill, G.W.: Researches in the lunar theory. In The Collected Mathematical Works of G.W. Hill, volume 1, pages 284-335. Carnegie Institution of Washington, (1905)

3. Heggie, D.C.: Escape in Hill's problem. In: Steves, B.A., Maciejewski, A.J. (eds.) The Restless Universe, pp. 109128, SUSSP Publications and Institute of Physics Publishing, Scotland and USA (2001)

4. Zotos, E.E., Steklain, A.F.: On the nature of the motion of a test particle in the pseudo-Newtonian Hill system. Astrophys. Space Sci. 364(10), 184 (2019)

5. Steklain, A.F., Letelier, P.S.: Stability of orbits around a spinning body in a pseudo-Newtonian Hill problem. Phys. Lett. A 373(2), 188-194 (2009)

6. Steklain, A.F., Letelier, P.S.: Newtonian and pseudoNewtonian Hill problem. Phys. Lett. A 352(4-5), 398-403 (2006)

7. Paczyńsky, B., Wiita, P.J.: Thick accretion disks and supercritical luminosities. Astron. Astrophys. 500, 203-211 (1980)

8. Chauvineau, B., Mignard, F.: Generalized Hill's problem: Lagrangian Hill's case. Celest. Mech. Dyn. Astron. 47(2), 123-144 (1990)

9. Papadakis, K.E.: The planar photogravitational Hill problem. Int. J. Bifurcation Chaos 16(6), 1809-1821 (2006)

10. Zotos, E.E.: Orbit classification in the Hill problem: I-the classical case. Nonlinear Dynam. 89(2), 901-923 (2017)

11. Batkhin, A. B.: Families of symmetric periodic solutions of the generalized Hill's problem. Keldysh Institute preprints, 60:24 pp, (2013)
12. Batkhin, A.B.: Web of families of periodic orbits of the generalized Hill problem. Dokl. Math. 90(2), 539-544 (2014)

13. Batkhin, A.B.: The search for periodic solutions with special symmetry in the Hill problem. Mat. Fiz. Komp'yut. Model. 22(3), 5-25 (2019)

14. Bruno, A.D.: The restricted 3-body problem: plane periodic orbits. Volume 17 of De Gruyter Expositions in Mathematics. Walter de Gruyter \& Co., Berlin (1994)

15. Hénon, M.: Numerical exploration of the restricted problem. V. Hill's case: Periodic orbits and their stability. Astron. \& Astrophys, 1:223-238, (1969)

16. Hénon, M.: Generating families in the restricted three-body problem, vol. 52 of Lecture Notes in Physics. New Series m: Monographs. Springer-Verlag, Berlin, (1997)

17. Batkhin, A.B.: Symmetric periodic solutions of the Hill's problem: I. Cosmic Res. 51(4), 275-288 (2013)

18. Morales-Ruiz, J.J.: Simó, C., Simon, S.: Algebraic proof of the non-integrability of Hill's problem. Ergodic Theory Dynam. Syst. 25(4), 1237-1256 (2005)

19. Morales-Ruiz, J.J.: Differential Galois theory and nonintegrability of Hamiltonian systems. Volume 179 of Progress in Mathematics. Birkhäuser Verlag, Basel (1999)

20. Morales-Ruiz, J.J., Ramis, J.-P.: Integrability of dynamical systems through differential Galois theory: a practical guide. In Differential algebra, complex analysis and orthogonal polynomials, volume 509 of Contemp. Math., pages 143220. Am. Math. Soc., Providence, RI, (2010)

21. Celletti, A.: Regularization Theory. Springer, Berlin (2010)

22. Celletti, A.: Basics of regularization theory. In: Steves, B.A., Maciejewski, A.J., Hendry, M. (eds.) Chaotic worlds: from order to disorder in gravitational N-body dynamical systems, pp. 203-230. Springer, Netherlands, Dordrecht (2006)

23. Ziglin, S.L.: Branching of solutions and the nonexistence of first integrals in Hamiltonian mechanics: I. Funct. Anal. Appl. 16(3), 181-189 (1982)

24. Audin, M.: Hamiltonian systems and their integrability, volume 15 of SMF/AMS Texts and Monographs. American Mathematical Society, Providence, RI; Société Mathématique de France, Paris, (2008)

25. Morales-Ruiz, J.J.: Peris, J.M.: On a Galoisian approach to the splitting of separatrices. Ann. Fac. Sci. Toulouse Math. 8(1), 125-141 (1999)

Publisher's Note Springer Nature remains neutral with regard to jurisdictional claims in published maps and institutional affiliations. 